\title{
Críticas ao "Tree Thinking": elucidando o significado das relações filogenéticas
}

\author{
Rafael Gomes de Souza
}

Universidade Federal do Rio de Janeiro, Museu Nacional, Programa de Pós-Graduação em Ciências Biológicas (Zoologia), Laboratório de Sistemática e Tafonomia de Vertebrados Fósseis, Quinta da Boa Vista, São Cristóvão 20940-040, Rio de Janeiro, Brasil. E-mail: rafelsouz@gmail.com

Souza R.G. (2017) Críticas ao "Tree Thinking”: elucidando o significado das relações filogenéticas. Pesquisa e Ensino em Ciências Exatas e da Natureza, 1(2): 115-130.

Resumo: O “Tree Thinking” é tido como a metodologia dominante na Biologia Sistemática atual. Todavia, críticas aos procedimentos executados pela mesma são diversas. Neste trabalho serão apresentadas e defendidas aquelas feitas por Fitzhugh no que tange a sua base filosófica e as consequências de tais modificações. Assim, o presente trabalho tem como objetivo demonstrar que o "Tree Thinking" é incompleto, por não reconhecer que as relações filogenéticas são do tipo causal, i.e., são hipóteses explanatórias, sumarizadas de forma prévia em um esquete explanatório (cladograma). Além disso, para embasar tal argumentação, será apresentada uma discussão sobre a definição e os objetivos da Biologia Sistemática e do "Tree Thinking”. Como resultado, será possível observar uma confusão entre classificar e sistematizar o conhecimento por aqueles que seguem o "Tree Thinking”. Ademais, o "Tree Thinking” falha na aquisição de explicações causais quanto à origem e fixação das características estudadas. Desta forma, o "Tree Thinking" pode ser considerado como uma prática incompleta dentro da Biologia Sistemática e, portanto, recomenda-se a aplicação das propostas de Fitzhugh.

Palavras chave: Biologia Sistemática, Cladística, Fitzhugh, Hennig, Sistemática Filogenética, Zimmerman.

"Tree Thinking" Criticism: elucidating the meaning of phylogenetic relationships

Abstract: The "Tree Thinking" is regarded as the dominant methodology in current Systematic Biology. However, criticisms of the procedures carried out by it are diverse. Here the criticisms made by Fitzhugh regarding its philosophical basis and the consequences of such modifications are presented and defended. Thus, the present work aims to demonstrate that "Tree Thinking", as it has been used, is incomplete because it does not recognize that phylogenetic relationships are of the causal type previously summarized in an explanatory sketch (cladogram). In addition, to support such an argument, a discussion on the definition and objectives of Systematic Biology and "Tree Thinking" is provided. As a result, it is possible to observe confusion between classifying and systematizing the knowledge by those who follow "Tree Thinking". In addition, "Tree Thinking" fails to provide causal explanations regarding the origin and fixation of the characteristics studied. In this way, "Tree Thinking" can be considered an incomplete practice within Systematic Biology and, therefore, the application of the proposals of Fitzhugh are recommended.

Key words: Systematic Biology, Cladistics, Fitzhugh, Hennig, Systematic Phylogenetics, Zimmerman.

“Consequentemente 'ciência' foi corretamente definida como 'a orientação sistemática do homem em seu ambiente” (Hennig 1966: 3 - tradução livre)

“Uma característica de qualquer ciência é a 'tarefa sem fim' e seu entendimento que o objetivo final provavelmente nunca será alcançado.” (Hennig 1966: 28 - tradução livre) 


\section{Introdução}

A Biologia Sistemática ${ }^{1}$ é uma área de pesquisa que, tradicional e resumidamente, tem como objetivo sistematizar os seres vivos (e.g., Hennig 1966), mas que, em uma era cladista, vem sendo tida como classificatória (e.g., Cantino \& de Queiroz 2006). Sua origem é incerta, mas podemos traçá-la com segurança desde os trabalhos de Aristóteles (e.g., Ferigolo 2015, 2016).

Em sua história a Biologia Sistemática se amparou em distintas bases filosóficas, teóricas e metodológicas, buscando a

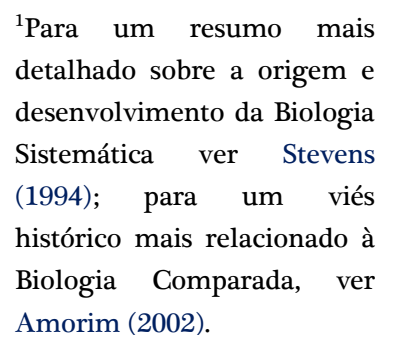

${ }^{1}$ Para um resumo mais detalhado sobre a origem e desenvolvimento da Biologia Sistemática ver Stevens (1994); para um viés histórico mais relacionado à Amorim (2002)

forma mais apropriada e natural de organizar os seres vivos. Alguns exemplos de escolas (sensu Amorim 2002): 1) Escola Lineana, fundada por Carolus Linnaeus (1735), que propunha uma hierarquização dos seres vivos baseadas em uma ideia essencialista (tal proposta foi adaptada ao que conhecemos hoje como os Códigos Internacionais de Nomenclatura Zoológica e Botânica); 2) "Catalográfica", uma escola não oficial, que buscava apenas catalogar as espécies sem preocupação quanto às suas causas; 3) Escola Fenética, que tem sua origem fundamentada no trabalho de Michener \& Sokal (1957), mas veja Sokal \& Sneath (1963), na qual se buscou organizar os seres vivos com base em fenogramas (diagramas ramificados que ilustram o grau de similaridade média entre os seres vivos); 4) Sistemática Gradista; embora os seguidores mais atuais concordem que a Sistemática Filogenética seja a forma mais apropriada para se estabelecer relações entre os seres vivos, os gradistas defendem que à classificação não deveria se restringir as relações filogenéticas e sim incluir conjuntos de características adaptativas, formando os grados (ver Darlington 1970; Mayr 1974; Ashlock 1974); e, 5) Sistemática Filogenética ou Cladística $^{2}$, originalmente formalizada por Hennig (1966), que estabelece um método para a proposição de relações filogenéticas valendo-se de matrizes graficamente similares às utilizadas pelos feneticistas, todavia, divergindo significativamente quanto ao conteúdo das mesmas, visto que os

${ }^{2}$ Mayr (1969) cunhou o termo Cladismo (do inglês Cladistics, o mesmo nome da renomada revista britânica) para se referir àqueles que seguiam as propostas de Hennig (para maior detalhamento desta história veja Williams \& Ebach (2008). Atualmente, em especial após suas atualizações / modernizações, principalmente quanto à estatística das análises, o termo Cladismo foi adotado como substituto para a Sistemática Filogenética, sendo, portanto reconhecidas como sinônimos. cladistas buscam preenchê-la com dados referentes às relações de homologias [sensu Nixon \& Carpenter (2012) ou homologias primárias sensu De Pinna (1991)] entre os organismos estudados; outra diferença significativa é a polarização evolutiva destas características (Nixon \& Carpenter 1993) dada na confecção dos diagramas, conhecidos como árvores filogenéticas ou cladogramas, sendo que a classificação deve representar, inequivocamente, as relações filogenéticas expressas por tais diagramas. Informações adicionais sobre as diferentes escolas, tais quais suas relações e desenvolvimento como ideias influenciadas pelo crescimento do conhecimento biológico e pela coesão social, podem ser encontrados em Christoffersen (1995). Podemos considerar que o advento da Sistemática Filogenética, proposta por Hennig (originalmente publicado em alemão, 1950 e posteriormente traduzido para inglês em 1966, e espanhol em 1968, representam um marco para a Biologia Sistemática, devido às propostas de padronização e modernização ante as teorias evolutivas de Darwin (1859). Em seu trabalho, Hennig $(1950,1966,1968)$ propõem um conjunto de métodos sustentados por bases filosóficas da época [e.g., Ziehen (1934), Hartmann 1933; para uma discussão sobre a base filosófica de Hennig, veja Rieppel (2016)] para aplicação da Sistemática Filogenética e pesquisas relacionadas (e.g., estudos populacionais, ontogenéticos) dentro do que reconhecemos como Biologia Sistemática.

Nas últimas décadas, com o advento da extração de dados moleculares dos organismos e o avanço em métodos analíticos específicos para tais dados (e.g., verossimilhança, análise bayesiana, métodos/programas de alinhamento e os inúmeros modelos evolutivos) podemos considerar que o cladismo adentra uma nova "Era" conhecida como genômica ou filogenômica 
(e.g., Wiens 2008). Esta nova vertente tem priorizado a utilização de dados moleculares em detrimento dos morfológicos, baseando-se em algumas supostas vantagens, como: 1) rapidez e facilidade na aquisição de dados e, 2) possiblidade de uma comparação mais fácil entre grupos bastante distintos, uma vez que todos os seres vivos possuem moléculas comparáveis (e.g., DNA, RNA), possibilitando um melhor entendimento acerca da "árvore da vida" (e.g., Simakov et al. 2015). Todavia, esforços metodológicos e teóricos são corriqueiros na literatura, buscando por uma visão concatenada entre o cladismo morfológico tradicional e seu expoente genômico (e.g., Wiens 2009; Giribet 2015; Lee \& Palci 2015). Tais novidades não se isentaram de críticas, as quais levaram atualmente a uma discussão acerca da eficiência dos três métodos analíticos em voga que são a "parcimônia, verossimilhança e análise bayesiana" (e.g., Goloboff 2003; Xu \& Pol 2003; O’Reilly et al. 2016).

Retomando o foco para o dito produto final da Sistemática Filogenética, temos os cladogramas (Hennig 1966). Os cladogramas são similares em sua retratação gráfica às árvores da vida, popularizadas por Darwin (1859) e Haeckel (1866) [veja Dayrat (2003) para um melhor entendimento da contribuição de Haeckel], cladogramas não possuem como função o estabelecimento de ancestralidade direta entre os organismos estudados (Christoffersen 1995), mas servem como representações gráficas para a hierarquização resultante de uma inferência filogenética (Hennig 1966). O’Hara (1997) cunhou a expressão “Tree Thinking” (pensamento baseado em árvores, numa tradução direta) para representar, de maneira didática, principalmente para estudantes de biologia, a importância e dependência de certas linhas de pesquisa às árvores filogenéticas ou cladogramas. O "Tree Thinking", baseado na ancestralidade em comum entre as espécies e na descendência com modificações, foi um ponto de mudança de paradigma na Biologia Sistemática quanto à sua forma de sistematização, sendo esta destinada ao reconhecimento de grupos naturais ou monofiléticos (Hennig 1966). Já em se tratando da base filosófica aplicada pelo "Tree Thinking", tem-se a vertente hipotético-dedutivista de Popper (1968, 1992) como cerne para nortear suas inferências e testes (e.g., De Queiroz \& Poe 2001; Farris et al. 2001). Todavia, nota-se que certos autores discordam do método hipotético-dedutivo e propõem bases distintas como preferíveis (e.g., Sober 1988; Rieppel 2003).

Como toda linha de pensamento científico, o "Tree Thinking" foi alvo de críticas. Dentre as mais comuns podemos ressaltar as voltadas para questões práticas como os métodos de inferência filogenética (e.g., Xu \& Pol 2003). Como críticas teóricas ao "Tree Thinking", temos aquelas relacionadas à forma de se interpretar e descrever as relações filogenéticas de um cladograma (e.g., Page \& Charleston 1998; Baum et al. 2005; Omland et al. 2008; Zachos 2016). Entre as críticas mais severas ao "Tree Thinking" estão as feitas por Fitzhugh (2005a, 2006a), enquadrando tanto aspectos práticos (i.e., críticas às técnicas de inferências filogenéticas) e teóricos (i.e., conhecimento prévio e aplicação de leis e teorias evolutivas na busca das explicações causais acerca da origem e fixação dos caracteres) e filosóficos (i.e., aplicação dos três tipos de raciocínio conforme proposto por Peirce (1878) nas etapas de proposição e teste das hipóteses).

O presente trabalho tem como objetivo demonstrar que o "Tree Thinking" é incompleto, por não reconhecer a causalidade natural das relações filogenéticas. Para embasar tal argumentação, uma discussão sobre a definição e objetivos da Biologia Sistemática será fornecida. Além disso, será apresentada uma definição geral do "Tree Thinking", baseado nas diferentes facetas observadas na literatura (i.e., as diferentes vertentes cladistas e afins). Buscando nortear a discussão, serão apresentadas e respondidas, de maneira contrastiva entre o "Tree Thinking" aqui definido e as propostas de Fitzhugh, as seguintes perguntas: 1) Queremos classificar ou sistematizar os seres vivos?; 2) O que queremos dizer por relações?; e, 3) Quais tipos de perguntas sobre relações são utilizados na Biologia Sistemática? E quais são suas respostas? Desta forma, será produzida uma avaliação da qualidade atual do "Tree Thinking", juntamente com sugestões e modificações para que ao fim seja apresentada uma linha de pensamento que melhor se enquadre nos objetivos da Biologia Sistemática, principalmente no que tange a aplicação do termo "relação filogenética". 


\section{Biologia Sistemática: Definição e Objetivos}

Do ponto de vista tradicional, a Biologia Sistemática pode ser dividida em Sistemática Filogenética e Taxonomia. A primeira buscaria estabelecer relações entre espécies distintas baseadas em teorias como descendência com modificação e ancestralidade em comum (Hennig 1966). A segunda teria como objetivo regulamentar e nomear espécies e seus respectivos grupos supra-específicos (Bertrand \& Härlan 2006; Cantino \& de Queiroz 2006). Atualmente, a Taxonomia é regrada por códigos internacionais, como, por exemplo, o Código Internacional de Nomenclatura Zoológica (CINZ) e o Código Internacional de Nomenclatura Botânica (CINB) que regulamenta a proposição de nomes para espécies, gêneros e famílias. Em contrapartida, o Phylocode (Cantino \& de Queiroz 2006) surge com o intuito principal de regulamentar a nomeação dos clados baseados em propostas filogenéticas. Sendo assim, a Biologia Sistemática teria como objetivos principais entender e propor hipóteses evolutivas para as espécies (Sistemática Filogenética) e, tendo tais informações como base, classificá-las (Taxonomia; e.g., de Queiroz \& Gauthier 1990, 1992, 1994; Sereno 1999, 2005).

Todavia, dois pontos devem ser elencados sobre tais objetivos da Biologia Sistemática. O primeiro ponto se refere à distinção entre Sistemática Filogenética e Taxonomia. Tal distinção tem como base principal a ideia de que o ato de confecção de hipóteses independe dos atos nomenclaturais/classificatórios (e.g., Christoffersen 1995). Embora Hennig traga em seu texto ambos os termos, Sistemática e Taxonomia, em sua escrita fica clara a ideia de que os atos descritivos e explanatórios (i.e., formulação de hipóteses) são inseparáveis ("assim, descrição e explicação não dividem as diferentes ciências umas das outras, mas em cada ciência e em cada disciplina parcial eles [descrição e explicação] estão interligados de uma forma inseparável" Hennig 1966: 3 - tradução livre). Tal qual Hennig, Zimmerman (ver Donoghue \& Kadereit 1992) também apontava como dependentes os atos nomenclaturais e suas explicações, sendo o primeiro relacionado ao segundo, i.e., as hipóteses de ancestralidade em comum e os passos de "transformação" dos caracteres (Zimmerman 1931). Desta forma, tal qual apontado por Fitzhugh (2008a), a sinonimização entre Sistemática e Taxonomia é desejável, no intento de minimizar confusões, principalmente no que se refere à ideia de que propor hipóteses e nomeá-las são etapas/procedimentos distintos.

O segundo ponto é que a Biologia Sistemática é muito mais que propor relações filogenéticas e hierarquizá-las no intuito classificatório. Em seu trabalho, Hennig (1966) defende a sistemática filogenética como padrão hierárquico universal para os estudos biológicos. Todavia o mesmo reconhece a existência de outros padrões hierárquicos que se relacionam e dependem uns dos outros como, por exemplo, as relações tocogenéticas e ontogenéticas, que podem ser hierarquizadas isoladamente por si mesmas, mas estão relacionadas entre si e subordinadas às relações filogenéticas. Esse complexo hierárquico foi nomeado por Hennig (1966) como relações hologenéticas (originalmente propostas por Zimmerman como espiral hologenética, termo utilizado para representar as relações continuas entre semaforontes e grupos de semaforontes) e que podem ser estudados isoladamente de maneira sistemática. Sendo assim, a Biologia Sistemática é uma área que não só busca sistematizar as hipóteses filogenéticas, como também lida com os outros níveis de hipóteses, i.e., relações hologenéticas (e.g., ontogenética e tocogenética). Fitzhugh (2008a, 2012) defende a pluralidade de níveis de hipóteses nas relações hologenéticas, incluindo as hipóteses perceptuais (aquelas que se referem às interpretações do sujeito). No presente trabalho, apresento quais são as perguntas contrastivas (de cunho causal), quais são relações (respostas às perguntas causais) e quais são representações (nível hierárquico correspondente) de cada uma delas (Tabela 1).

Hennig (1966) defende como objetivo, não só da Biologia Sistemática, mas de quaisquer outras ciências naturais, a apresentação completa e exaustiva de uma explicação (causal) sobre um evento que seja limitado temporalmente. Neste contexto, entende-se por evento nas ciências naturais não só os processos causais do mesmo, mas também sua entidade física e individualizada correspondente. De forma mais detalhada, Fitzhugh (2008a) atribui à Biologia Sistemática o objetivo de obter entendimento causal sobre as propriedades presentes nos 
organismos em diferentes estágios de sua história de vida ou compartilhada por outros indivíduos. Desta forma, podemos resumir o objetivo da Biologia Sistemática como o de explicar causalmente os caracteres observados na forma de hipóteses referentes às relações hologenéticas de Hennig $(1966,1965)$ e Fitzhugh (2008a), sendo fornecidos nomes tanto para as hipóteses isoladas (e.g., espécies) quanto para o conjunto de hipóteses (e.g., clados), para, por fim, sistematizá-los de forma hierárquica.

Tais objetivos são condizentes com os três níveis de explicações propostas por Mayr (1961), que são as explicações descritivas e as causais (próximas e últimas), veja Beatty (1994), Laland et al. (2011) e Fitzhugh (2012) para melhor entendimento da contribuição de Mayr às bases filosóficas da Biologia Sistemática. São também congruentes com os objetivos propostos por Hempel (1965) para as ciências como um todo. Portanto, o presente trabalho assumirá a definição de Biologia Sistemática inicialmente trabalhada por Hennig (1966) e atualizada por Fitzhugh (2008a).

Tabela 1. Sumarização das perguntas causais, respostas e representação de cada nível de hipótese incluída nas relações hologenéticas (modificado de Fitzhugh 2008a).

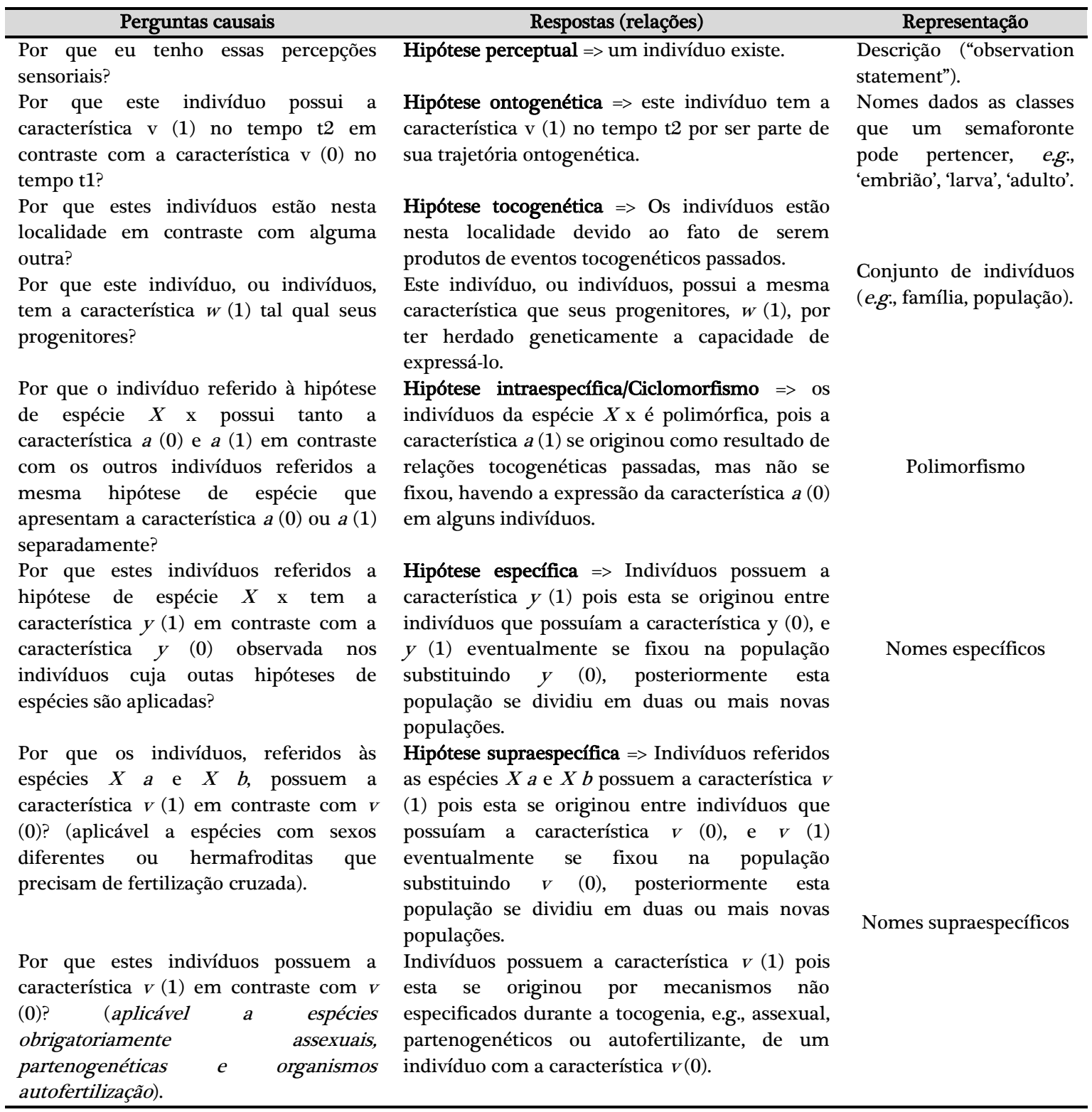




\section{As diferentes faces do "Tree Thinking"}

Desde o advento da Sistemática Filogenética até os dias da Cladística atual, podemos reconhecer vertentes distintas de estudo que priorizam ou aplicam o "Tree Thinking”. Aqui serão apresentadas e discutidas as principais delas. Para termos classificatórios, o presente trabalho considera como vertentes do "Tree Thinking" todas aquelas que consideram o cladograma como produto final da pesquisa, cuja topologia é o principal resultado da análise e os caracteres são as evidências utilizadas para a formulação de tais relações. Além disso, é importante ressaltar que o cladograma no "Tree Thinking" tem um contexto hierárquico que deve ser usado na classificação dos organismos estudados.

As primeiras proposições que embasaram o "Tree Thinking" foram as inicialmente propostas por Zimmerman (1931) [mas também veja Donoghue \& Kadereit (1992)] que defendem que a melhor forma de agrupar os organismos é a filogenética, pois entre as opções disponíveis, esta possibilita o reconhecimento de grupos naturais resultantes do agrupamento genealógico dos organismos. Hennig $(1965,1966)$ não só defende as proposições de Zimmerman como as aprimora, fornecendo critérios para designação dos grupos classificatórios baseados em grupos monofiléticos, ressaltando a importância da heterobatimia nos eventos de especiação.

Como ilustrado por Christoffersen (1995), embora inúmeras diferenças possam ser apontadas entre as vertentes atuais do cladismo (e.g., Farris 2013), todas derivam de uma mesma proposta inicial (Zimmerman + Hennig). Desta forma, podemos exemplificar alguns pontos de divergência entre elas: 1) forma de proposição e reconhecimento das homologias (e.g., De Pinna 1991 contra Nixon \& Carpenter 2012); 2) os métodos de análise destes dados (e.g., Williams \& Ebach 2008 contra Farris 2013); e, 3) na adoção previa à análise de uma base teórica (e.g., Hennig 1966 contra Brower 2000). Há de se convir que, mesmo com tais divergências, em todas essas vertentes entende-se que: 1) as relações filogenéticas são a representação das relações genealógicas das diferentes espécies; 2) o objetivo da Sistemática Filogenética é hierarquizar e classificar os seres vivos por meio do estabelecimento das relações filogenéticas sumarizadas no cladograma e reconhecimento dos grupos monofiléticos; e, 3) para a descoberta de tais relações filogenéticas (produção do cladograma) são utilizados caracteres (de quaisquer fontes) para a identificação de sinapomorfias, que são, juntamente com a hetorabatimia, as evidências necessárias para o reconhecimento de grupos monofiléticos.

São incluídos no Cladismo outros tipos de análise para a produção de árvores, como o likelihood (verossimilhança; e.g., Felsenstein 1981, 2004; Swofford et al. 1996; Haber 2011) e a análise bayesiana (e.g., Huelsenbeck et al. 2001; Huelsenbeck \& Ronquist 2001; Archibald et al. 2003). Estes métodos também poderiam ser enquadrados no "Tree Thinking" aqui proposto, embora existam diferenças sensíveis na metodologia de análise (e.g., o fato de serem afirmações de verdade baseadas em modelo a posteriori sobre divergência filogenética), por buscarem como resultado final a produção de um dendograma que represente as relações filogenéticas entre as espécies estudadas ${ }^{3}$. Todavia, no presente trabalho, não será apresentada mais nenhuma discussão sobre a verossimilhança e a análise bayesiana, visto que original e historicamente estes não foram produzidos no intuito de gerar hipóteses e sim para atestar a plausibilidade revisada de hipóteses já estabelecidas (para maior detalhamento sobre tal discussão ver Fitzhugh 2006a, 2012, 2014). Desta forma, pode-se considerar o Likelihood (verossimilhança) e a bayesiana, no Cladismo, como adaptações metodológicas equivocadas oriundas de outras áreas.

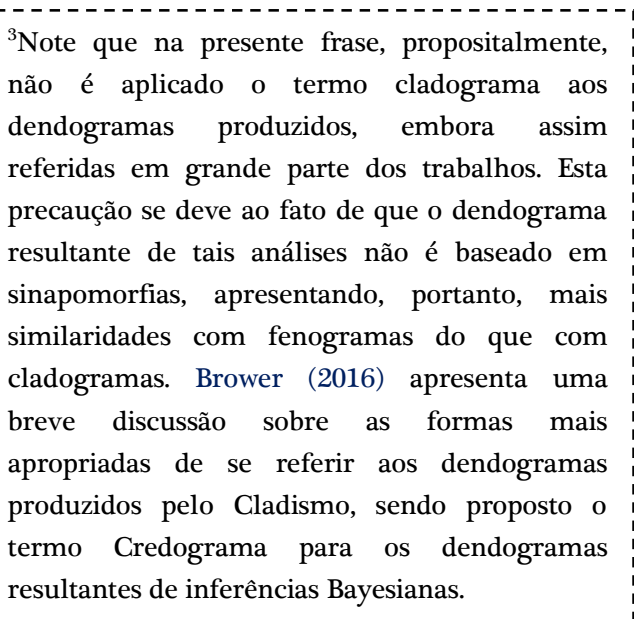
Note que na presente frase, propositalmente, não é aplicado o termo cladograma aos dendogramas produzidos, embora assim referidas em grande parte dos trabalhos. Esta precaução se deve ao fato de que o dendograma resultante de tais análises não é baseado em sinapomorfias, apresentando, portanto, mais similaridades com fenogramas do que com cladogramas. Brower (2016) apresenta uma breve discussão sobre as formas mais apropriadas de se referir aos dendogramas produzidos pelo Cladismo, sendo proposto o termo Credograma para os dendogramas resultantes de inferências Bayesianas.

Todavia, existe uma vertente da Sistemática Filogenética que se desvencilhou do "Tree Thinking” e, portanto, não se enquadra nas características delimitadoras aqui propostas. Esta 
outra vertente foi proposta e defendida por Fitzhugh (2005a, 2006a) e tem em sua base Hennig (1966), principalmente em relação à sua sistematização e às suas relações hologenéticas, mas as atualiza ante o pragmatismo norte-americano de Charles Sanders Peirce (e.g., 1878). Nesta última vertente destaca-se que tanto a Biologia Sistemática, como um todo, quanto a Sistemática Filogenética se enquadram no padrão das inferências abdutivas (e.g., Fitzhugh 2005a, 2006a; veja Tabela 2 para melhor entendimento acerca dos tipos de inferências), e baseando-se nesta mudança de paradigmas Fitzhugh (2005b, 2006b, 2006c, 2008a, 2008b, 2009, 2012, 2016a, 2016b, 2016c, 2016d) demonstra os impactos de sua proposta em todos os níveis relacionados à forma com que o Cladismo vem sendo feito.

Portanto, no intuito de destacar as diferenças que serão debatidas e tornar mais didática as discussões, o termo "Tree Thinking", como definido no início desta sessão, abrangerá, de uma forma bastante simplificada, vertentes consideradas concorrentes e que partilham de bases, princípios e métodos distintos. No entanto, mesmo ante essas diferenças, elas podem ser enquadradas dentro do "Tree Thinking", por compartilharem o mesmo objetivo e algumas outras características discutidas previamente e resumidas na Tabela 3. Além disso, as vertentes agrupadas sob a alcunha do "Tree Thinking" divergem de maneira similar às da vertente da Sistemática Filogenética proposta e defendida por Fitzhugh (2005a, 2006a).

Tabela 2. Sumário das características e diferenças acerca dos três tipos principais de inferências, baseado no trabalho de Fitzhugh (2005a, 2006a).

\begin{tabular}{llll}
\hline Dedução & \multicolumn{2}{c}{ Indução } & Abdução \\
\hline Aplicação de regras gerais a casos & As premissas pertencem a uma & Contexto onde hipóteses são \\
particulares. No entanto, a inferência & mesma classe, o que permite uma & formuladas & e \\
dedutiva é incapaz de gerar uma & conclusão expansiva acerca de objetos & posteriormente testadas no \\
conclusão que contenha mais & não observados ou eventos futuros & contexto da justificação, \\
informações do que as já contidas & relacionados a classe cujas premissas sendo, portanto, a & a \\
nas premissas. Utilizada para & pertençam. Devido à capacidade ferramenta fundamental \\
fornecimento de evidências de teste & ampliativa é uma inferência para iniciar o processo de \\
de uma hipótese/teoria. & associada com o teste de hipóteses. & pesquisa científica. \\
\hline
\end{tabular}

Tabela 3. Classificação das vertentes de Sistemática Filogenética/Cladismo quanto à sua compatibilidade o “Tree Thinking” aqui definido.

\begin{tabular}{|c|c|c|}
\hline & "Tree Thinking" & Sistemática Filogenética (Fitzhugh) \\
\hline Objetivos & $\begin{array}{l}\text { Estabelecer as relações filogenéticas entre } \\
\text { os organismos estudados. }\end{array}$ & $\begin{array}{l}\text { Explicação causal das similaridades } \\
\text { compartilhadas entre os indivíduos } \\
\text { de diferentes espécies. }\end{array}$ \\
\hline Teoria base & $\begin{array}{l}\text { Nenhuma ou Ancestralidade em comum } \\
\text { e Descendência com modificação. }\end{array}$ & $\begin{array}{l}\text { Ancestralidade em comum e } \\
\text { Descendência com modificação. }\end{array}$ \\
\hline Caracteres & $\begin{array}{l}\text { Base para produção de cladogramas } \\
\text { sendo considerados como causa das } \\
\text { relações filogenéticas encontradas. }\end{array}$ & $\begin{array}{l}\text { Elementos a serem explicados } \\
\text { causalmente pelas hipóteses de } \\
\text { relações filogenéticas que melhor } \\
\text { expliquem o conjunto estudado. }\end{array}$ \\
\hline Produto Final & $\begin{array}{l}\text { Cladograma que servirá de base para } \\
\text { classificação dos organismos estudados. }\end{array}$ & $\begin{array}{l}\text { Explicação sobre origem e fixação } \\
\text { dos caracteres } \quad \text { estudados, }\end{array}$ \\
\hline & & $\begin{array}{l}\text { inicialmente explicadas pelas } \\
\text { relações filogenéticas em um } \\
\text { esquete explanatório (cladograma). }\end{array}$ \\
\hline Exemplos de Autores & $\begin{array}{l}\text { Zimmerman (1931, 1943, 1953); Hennig } \\
\text { (1965, 1966); Platnick (1979); Farris } \\
\text { (1983); Patterson (1994); Swofford et al. } \\
\text { (1996); Brower (2000); Kitching et al. } \\
\text { (1998); Assis (2009). }\end{array}$ & Fitzhugh (2005a, 2006a). \\
\hline
\end{tabular}




\section{Queremos classificar ou sistematizar os seres vivos?}

Antes de responder tal pergunta devemos definir o que entendemos por classificar e sistematizar. O presente trabalho se baseia nas seguintes definições propostas por O'Hara (1993), Malner \& Bunge (1997) e Bunge (1998), aplicadas por Fitzhugh (2008a):

Classificação: agrupar objetos com base em uma ou mais características específicas.

Sistematização: organização de observações em um sistema de conceitos, na forma de hipóteses, de acordo com uma ou mais teorias.

Complementarmente, Griffths (1974) defende a sistemática como o ordenamento de coisas de acordo com as relações dos elementos/sistemas, ou seja, o ordenamento é baseado nas relações inferidas (e.g., hipóteses) derivadas de princípios estabelecidos (e.g., teorias). Classificar é a atividade lógica de ordenar conceitos dentro de classes, sendo classes equivalentes às relações dos elementos/sistemas ordenadas sistematicamente (e.g., hipóteses).

A Sistemática Filogenética sensu Hening (1966) considera que as relações filogenéticas devem ser representadas na forma de sistemas hierárquicos, portanto, por atos de sistematização. Por sistematização Hening (1966) entende não uma simples descrição das coisas, fenômenos ou processos e sim o ordenamento delas, levando também em consideração as explicações das mesmas. Todavia, essa ideia de sistematização se perdeu na própria aplicação da Sistemática Filogenética por Hening (1966), que culminou no simples ato de produção de cladogramas e na designação de nomes para os grupos monofiléticos, adquirindo, portanto, um viés puramente classificatório ("Tree Thinking"). Sendo assim, Hening (1966) conseguiu reconhecer a importância do viés da sistematização na ciência e principalmente na Biologia, mas falhou em sua aplicação, como as demais vertentes derivadas dele, que estão contidas no "Tree Thinking".

Alguns defensores do "Tree Thinking" propõem que o cladismo, em geral, independe de teorias, principalmente das evolutivas [i.e., Brower (2000) para "aplicação" do cladismo sem bases teóricas), justificando tal pensamento por interpretações equivocadas das propostas de Popper, como, por exemplo, nos processos de falseamento (ver Fitzhugh 2016a). Esse ponto de vista cladístico é condizente com propostas de cunho classificatório apenas e, portanto, inicialmente válido como instrumento classificatório. Além disso, o "Tree Thinking" se assemelha bastante com as ideias expostas no CINZ, CINB e no Phylocode, códigos estes que têm como proposta serem classificatórios. No entanto, o cladismo, como uma derivação da Sistemática Filogenética deveria, pelo menos a priori, como parte da Biologia Sistemática, e por derivar das propostas teóricas de Hennig (1966), ter em sua essência um viés de sistematização buscando assim explicações causais para os objetos de estudo e organizando-os de acordo com as principais teorias aplicadas à Biologia Sistemática (i.e., descendência com modificação e ancestralidade em comum).

Ressalto que o ponto de vista do "Tree Thinking”, em que inferências filogenéticas podem ser realizadas de forma independente de quaisquer teorias, é necessariamente uma conclusão equivocada, que deve ser evitada (veja Fitzhugh 2008a, 2010). Primariamente, um dos principais objetivos do trabalho de Hennig (1966) foi justamente formalizar um conjunto de práticas e métodos para estudos na Biologia Sistemática. Complementarmente, havia-se também

${ }^{4}$ Como originalmente reforçado por Hennig (1966: 3 - tradução livre): “Coisas, fenômenos e processos são aqui designados como 'sistemáticos' e não devem ser entendidos como uma simples descrição de um fenômeno isolado, mas como um elemento de uma ordem de coisas, fenômenos ou processos de acordo com leis. Consequentemente, 'ciência' foi corretamente definida como 'a orientação sistemática do homem em seu ambiente."

${ }^{5}$ Zimmerman $(1931,1937,1943,1953)$ já trazia em seus trabalhos essa demanda sistemática e forneceu o aporte teórico e metodológico que foi base para a proposta de Hennig (1966), veja Donoghue \& Kadereit (1992) para maiores detalhes. demanda por regularizar e formalizar o conjunto teórico aplicado por tal área para que a mesma pudesse ser equiparada às demais ciências e pudesse desbancar as propostas concorrentes que, em geral, eram incompletas e mal fundamentadas ${ }^{4,5}$. Além disso, em termos metodológicos, os 
defensores do "Tree Thinking” (ver Swofford et al. 1996; Page \& Holmes 1998; Brower 2000; de Queiroz \& Poe 2001; Farris 2008 e referências ali citadas) alegam que é apenas necessária a parcimônia [sic.] para a confecção dos cladogramas (veja Fitzhugh 2006a, 2012, para melhor compreensão do mau uso da parcimônia). Todavia o princípio teórico mais aplicado para o enraizamento e polarização das características é o do grupo externo. Este princípio necessariamente invoca as teorias de ancestralidade em comum e descendência com modificação para organização das explicações sobre as similaridades observadas (veja Fitzhugh 2006b). É importante ressaltar que o princípio do grupo externo também é usado para a polarização a posteriori dos caracteres nas árvores recuperadas (Nixon \& Carpenter 1993). Sendo assim, o grupo externo possui duas funções diferentes, porém complementares, na Sistemática Filogenética: 1) assume uma função de contraste nas "why-questions" (Fitzhugh 2006b); 2) atua na polarização a posteriori dos caracteres nas árvores recuperadas (Nixon \& Carpenter 1993).

Como explicitado na teoria de Hennig (1966), e aprimorado por Fitzhugh (2008a), não só a Biologia Sistemática, mas também os métodos de Sistemática Filogenética buscam propor e aplicar nomes às hipóteses explanatórias fornecidas a um conjunto de semaforontes (seja este um conjunto pertencente a qualquer uma das classes hologenéticas de Hennig, mas também veja Fitzhugh (2008a, 2009), e.g., ontogenética, específica, filogenética) inferidas a partir de um conjunto identificado de teorias (e.g., teorias de ancestralidade em comum e descendência com modificação). Portanto, o ato nomenclatural na Biologia Sistemática não consiste apenas em classificar, segregando e identificando indivíduos, e sim também em sistematizar suas hipóteses, provendo nomes que facilitem a comunicação e sumarizem o conteúdo de tais hipóteses (Fitzhugh 2008a).

Desta forma, a prática do "Tree Thinking" de atribuir nomes aos conjuntos de semaforontes (e.g., Homo sapiens, Hominidae) identificados por meio de suas autapomorfias, i.e., de sua diagnose, não satisfaz os pré-requisitos de sistematização imbuídos na Biologia Sistemática. Portanto, o "Tree Thinking" é uma prática apenas classificatória, que não tem por objetivo e nem a função de fornecer e organizar o entendimento causal das percepções estudadas como requerido nas práticas da Biologia Sistemática.

\section{O que queremos dizer por relações?}

Estabelecido que o objetivo da Biologia Sistemática é sistematizar o conhecimento biológico e não apenas classificá-lo, devemos agora entender o significado do termo 'relações' na Biologia Sistemática.

Como proposto por Coffey (1938), o conceito objetivo de relação estabelece uma unidade conceitual entre um par de coisas no domínio de alguma outra categoria. Para o estabelecimento de uma relação real é necessária a presença de dois ou mais sujeitos, uma fundação e um termo individual distinto dos sujeitos. O termo é responsável por fornecer a unidade conceitual (relação) entre os sujeitos por meio do fundamento (e.g., causa, similaridade). Coffey (1938) ainda pontua a existência de dois tipos de relações: as de similaridade (relações de mesma denominação) e as de diferença (relações de diferentes denominações). As relações de similaridade são estabelecidas quando sujeitos diferentes são atribuídas a uma mesma categoria (i.e., espécimes da mesma espécie) por compartilharem alguma propriedade em comum (i.e., detecção de características diagnósticas). As relações de diferença ou causalidade são estabelecidas entre sujeitos de categorias diferentes (i.e., espécimes de espécies diferentes) por meio do estabelecimento de relações de causa-efeito (i.e., a hipótese de que o gênero A é a causa dos efeitos apresentados pelas espécies $A$ a e $A b$ ).

Para Zimmerman (Donoghue \& Kadereit 1992), relações filogenéticas são proposições comparativas em que o grau de relacionamento entre duas espécies distintas é maior quando estas compartilham um ancestral mais recente em comum do que com uma terceira espécie. Por meio do estabelecimento de tais relações e das diferenças nos graus de relacionamento, Zimmerman (Donoghue \& Kadereit 1992) estabelece seu critério de hierarquização, que embora assumidamente arbitrário quanto ao grau de inclusão de espécies em cada grupo, deve ser 
baseado no compartilhamento de um ancestral em comum mais recente entre as espécies ali incluídas. Este critério de hierarquização foi o mesmo adotado por Hennig $(1965,1966)$, que o aprimorou por meio do conceito de grupos monofiléticos. Além disso, relações filogenéticas para Hennig (1966) são, além da definição já dada por Zimmerman, o reconhecimento de relações genealógicas via conexões tocogenéticas entre os organismos (teoria da ancestralidade em comum).

Embora possamos reconhecer critérios de causalidade embutidos na definição de relações usadas por Zimmerman e Hennig, provenientes da ancestralidade em comum (genealogia), podemos considerar tais relações, seguindo as definições de Coffey (1938), como relações de similaridade. Apesar da genealogia poder possuir um viés causal ela é aplicada como um critério puramente relacional (A é mais próximo de $\mathrm{B}$ do que de $\mathrm{C}$ pelo fato do ancestral $\mathrm{AB}$ ser mais próximo de $\mathrm{A}$ e $\mathrm{B}$ do que do ancestral de $\mathrm{ABC}$ ) sendo utilizada como um detector de graus de similaridade.

No "Tree Thinking", o termo relações tem seu uso proposto como correspondência de similaridade onde espécies são mais proximamente vinculadas umas às outras por meio de seu posicionamento topológico, implicando o compartilhamento de um ancestral comum (e.g., Cantino \& de Queiroz 2006; clado X inclui todas as espécies mais proximamente relacionadas às espécies A e B do que à espécie C). O Phylocode (Cantino \& de Queiroz 2006) pode ser considerado, pelos cladistas, o auge de tal tipo de uso do termo relações em Biologia Sistemática.

Em contrapartida, Fitzhugh reconhece que ambos os tipos de relações são importantes para a Biologia Sistemática. Ele propõe que as relações de similaridade são utilizadas no estabelecimento de caracteres compartilhados, enquanto que as relações de diferença estabelecem relações de causa-efeito (e.g., hipóteses de espécies, hipóteses filogenéticas). Desta forma, embora os defensores do "Tree Thinking" apliquem o termo relações no contexto das similaridades, ele deveria também possuir a conotação de relações de diferença, que implica em uma relação de causa-efeito (i.e., a espécie A se relaciona com a espécie B e compõem o clado C, por compartilharem um ancestral comum mais recente que explica a origem e fixação dos caracteres compartilhados entre estas espécies). Vale ressaltar que as relações de similaridade são importantes tanto para a proposição de homólogos $^{6}$ (sensu Owen 1847; ver Fitzhugh 2006b) quanto para o reconhecimento de novos espécimes para espécies já conhecidas (i.e., espécimes estes que não tenham sido utilizados como tipos na proposição da hipótese de espécie). Já as relações de causa-efeito são importantes para o fornecimento das explicações iniciais quanto à origem e fixação das similaridades, como hipóteses de homólogos, compartilhadas entre os indivíduos de diferentes espécies (Fitzhugh 2005, 2006a).

Para via de esclarecimentos, o presente trabalho
segue a proposta de Fitzhugh (2006a,b) que é
considera, portanto, homóloga como um nível de
hipótese distinta da homologia (conclusão similar
foi proposta por Williams \& Ebach (2012). Desta
forma as hipóteses de homólogos se referem às
similaridades estabelecidas entre características
de organismos distintos. As hipóteses de
homologia (sensu Owen 1847) devem ser
abandonadas e substituídas pelas hipóteses de
homogenia e homoplasia (sensu Lankester 1870).
Sendo que as hipóteses de homogenia e
homoplasia são as explicações causais quanto à
origem e fixação das hipóteses de homólogos.
${ }^{6}$ Para via de esclarecimentos, o presente trabalho hipótese distinta da homologia (conclusão similar foi proposta por Williams \& Ebach (2012). Desta forma as hipóteses de homólogos se referem às homologia (sensu Owen 1847) devem ser abandonadas e substituídas pelas hipóteses de (sensu Lankester 1870). homoplasia são as explicações causais quanto à

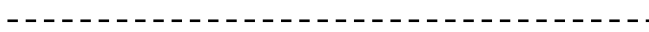

Baseado nos objetivos da Biologia Sistemática discutidos anteriormente, pode-se observar que o uso de relações no "Tree Thinking" é insatisfatório e no mínimo incompleto, por se restringirem à utilização apenas das relações de similaridades, ignorando o efeito causal que as relações filogenéticas possuem. Todavia, a vertente atualizada por Fitzhugh $(2005,2006 \mathrm{a})$ traz consigo o aporte sistemático requerido, utilizando os dois tipos de relações aqui discutidas, com ênfase especial para as relações de causa-efeito (diferença) inicialmente estabelecidas pelas relações de ancestralidade em comum e descendência com modificação.

\section{Quais tipos de perguntas sobre relações são utilizados na Biologia Sistemática? E quais são suas respostas?}

Zimmerman (1937) [veja Donoghue \& Kadereit (1992)] argumenta que o entendimento da história de vida dos organismos só é possível respondendo às seguintes perguntas: 1) Quais 
são os efeitos resultantes da evolução?; 2) Qual foi o curso histórico da filogenia?; e, 3) Quais são os fatores causais responsáveis pela filogenia? Como resposta à primeira pergunta ele propõe as descrições referentes aos organismos estudados; portanto, propõe caracteres e estabelece os homólogos. Para a segunda pergunta, ele considera dois objetivos, um 'próximo' ('taxon phyletics") e um 'distante' ("character phyletics"). O primeiro se refere à construção de topologias, ou seja, à proposição de ancestrais comuns para os organismos estudados; o segundo objetivo refere-se à evolução, i.e., à origem e fixação dos caracteres. Este segundo objetivo é considerado por Zimmerman (1937) como sendo a base para todo o curso histórico da filogenia. Os fatores causais da evolução (terceira pergunta) são as proposições propriamente ditas para as hipóteses filogenéticas, que complementam os efeitos da evolução (segunda pergunta).

Hennig (1966) absorve tais questionamentos e métodos de resposta em suas discussões teóricas, embora não os aplique de forma completa. Desta forma, no "Tree Thinking", apenas perguntas referentes ao curso histórico da filogenia foram trabalhadas (e.g., Felsenstein 2004). Podemos, portanto, nos restringir a duas questões: 1) qual a história evolutiva desse clado?; e, 2) como foi a evolução da característica X? Em geral, as respostas para à primeira pergunta são restritas à descrição da topologia recuperada (e.g., Brochu 2013). No que se refere à segunda pergunta (apesar de ser um tanto quanto mais rara em trabalhos cladísticos), sua resposta versará sobre o mapeamento de características na topologia encontrada, estando esta feição presente ou não na lista de caracteres (e.g., Joshi \& Edgecombe 2017), sem apresentar os contextos de origem e fixação de tal característica [veja Fitzhugh (2014) para críticas ao mapeamento de caracteres].

Fitzhugh (2005a, 2006a, 2008a), ao reconhecer a Biologia Sistemática como essencialmente abdutiva, atribui importância às "why-questions" na estruturação do raciocínio. Neste contexto, as "why-questions" possuem uma estrutura contrastiva em que indivíduos que apresentam características $\mathrm{A}^{7}$ são contrastados com indivíduos que apresentam a característica $\mathrm{B}$, sendo A e B homólogos (Fizthugh 2006a,b, 2016a,b). Este tipo de questionamento deve ser realizado para cada coluna (caráter) da sua matriz de dados (Fitzhugh 2006) resultando em uma série de perguntas que podem ser equiparadas com as perguntas dois e três de Zimmerman. Suas respostas serão referentes à origem e fixação dos caracteres envolvidos e também às hipóteses de "splits" populacionais, sendo ambas apenas inicialmente explicadas pelo cladograma (esquete explanatório; Fitzhugh 2006a, 2012). Outro ponto de extremo valor nesta vertente é o reconhecimento de outros tipos de perguntas, que não se restringem apenas ao nível filogenético, mas se aplicam a todos os níveis hologenéticos propostos por Henning (ver Fitzhugh 2008).

${ }^{7}$ No cladismo a distinção entre caractere e estado de caráter é tido como algo fundamental (e.g., Sereno 2007). No entanto, no presente trabalho caractere será utilizado como definido por Fitzhugh (2006b: 263 - tradução livre), "uma proposição de sujeito-predicado que se refere especificamente ao estado de ser de um objeto", portanto, sem a distinção entre caractere e estado de caráter. Esta definição proposta por Fitzhugh, em que caractere é a fusão do caractere mais um dos estados de caráter cladísticos, não deve ser confundida com "character statement" proposto por Sereno: o caractere mais todos seus estados de caráter.

Portanto, diante dos objetivos da Biologia Sistemática, a proposta de Fitzhugh abarca as proposições originais e as complementa, aproximando seus métodos e resultados ao que é buscado. Já o "Tree Thinking" apresenta uma limitação muito grande quanto aos objetivos cumpridos por seus métodos, sendo em geral restrita apenas à proposição de ancestrais em comum, ou seja, somente lida com respostas relacionadas à pergunta dois, com enfoque na topologia geral, desconsiderando a origem e fixação dos caracteres.

\section{Conclusões}

Baseado na discussão aqui apresentada, conclui-se que o "Tree Thinking" apresenta fortes incoerências quanto aos objetivos da Biologia Sistemática, tornando tal método e pensamento no mínimo incompletos diante dos pré-requisitos científicos. Ainda, os pensamentos oriundos da Sistemática Filogenética propostos por Zimmerman e Hennig não deveriam ser abandonados, muito menos menosprezados. Considerando os objetivos da Biologia Sistemática, 
tais proposições são as que mais se aproximam em cumpri-los. Não menos importante, o reconhecimento da Biologia Sistemática como uma ciência histórica abdutiva em sua essência não só favorece as proposições originais, como as aprimoram para melhor cumprir seus objetivos como ciência.

Neste contexto, uma última pergunta pode ser feita: buscamos relações entre as espécies ou explicações para os caracteres? Dentro dos parâmetros aqui apresentados como objetivos da Biologia Sistemática, a resposta é buscar a explicação para os caracteres (que compõem as "whyquestions" do trabalho). As relações entre as espécies se tornam um subproduto das inferências filogenéticas, servindo como base inicial para a proposição das explicações causais na forma de hipóteses sobre a origem e fixação dos caracteres. Desta forma, o "Tree Thinking” pode ser considerado incompleto em comparação com as proposições de Fitzhugh. Este autor se preocupa com as relações entre as espécies, sendo os caracteres apenas dados a serem computados para revelar o grau de relacionamento entre os organismos estudados. No intuito de sumarizar a incompletude do "Tree Thinking" a Tabela 4 contrasta, resumidamente, os objetivos da Biologia Sistemática, como ele é aplicado no "Tree Thinking” em Fitzhugh.

Portanto, tal qual Hennig (1965) considerou a Sistemática Filogenética um aprimoramento em consequência aos conhecimentos da era morfológica de sistematização, o presente trabalho considera as proposições de Fitzhugh como um aprimoramento teórico e metodológico das vertentes do "Tree Thinking” (Sistemática Filogenética/Cladística).

Tabela 4. Contraste metodológico das bases do "Tree Thinking" e das proposições de Fitzhugh para atingir os objetivos da Biologia Sistemática.

\begin{tabular}{lcc}
\multicolumn{1}{c}{ Biologia Sistemática } & “Tree Thinking” & Sistemática Filogenética (Fitzhugh) \\
\hline Relações de similaridade & Super valorizado & Incluso \\
Relações de diferença (Causalidade) & Raro & Incluso \\
Hierarquização via & Classificação & Sistematização \\
Reconhecimento de diferentes níveis de hipótese & Raro & Incluso \\
\hline
\end{tabular}

\section{Agradecimentos}

Gostaria de agradecer ao Dr. Rodrigo Giesta Figueiredo (Universidade Federal do Espirito Santo) pelo incentivo e apoio quanto ao desenvolvimento deste trabalho; a Kamila L.N. Bandeira (Museu Nacional/Universidade Federal do Rio de Janeiro) e Wanderson Dutra Vittorazzi pelos comentários e auxílio nas primeiras versões deste manuscrito; Kirk Fitzhugh (Natural History Museum, Los Angeles) e Helio Ricardo da Silva (Universidade Federal Rural do Rio de Janeiro) pelos auxílios e discussões no que concerne a parte filosófica do trabalho.

\section{Referências}

Amorim D.S. (2002) Fundamentos de Sistemática Filogenética. Ribeirão Preto: Holos. 156 p.

Archibald J.K., Mort M.E. \& Crawford D.J. (2003) Bayesian inference of phylogeny: a nontechnical primer. Taxon, 52: 187-191.

Ashlock P.D. (1974) An evolutionary systematist's view of classification. Systematic Zoology, 28(4): 441-450.

Assis L.C.S. (2009) Coherence, correspondence, and the renaissance of morphology in phylogenetic systematics. Cladistics, 25: 528-544.

Baum D.A, Smith, S.D \& Donovan S.S.S. (2005) The Tree-Thinking Challenge. Science, 310: 979980.

Beatty J. (1994) The proximate/ultimate distinction in the multiple careers of Ernst Mayr. Biology \& Philosophy, 9: 333-356.

Bertrand Y. \& Härlan M. (2006) Stability and universality in the application of taxon names in phylogenetic nomenclature. Systematic Biology, 55: 848-858. 
Brochu C.A. (2013) Phylogenetic relationships of Palaeogene ziphodont eusuchians and the status of Pristichampsus Gervais, 1853. Earth and Environmental Science Transactions of the Royal Society of Edinburgh, 103: 521-550.

Brower A.V.Z. (2000) Evolution is not a necessary assumption of cladistics. Cladistics, 16(1): 143154.

Bunge M. (1998) Philosophy of science, volume 1, from problem to theory. New Brunswick: Transaction Publishers. $624 \mathrm{p}$.

Cantino P.D. \& de Queiroz K. (2006) PhyloCode: International code of phylogenetic nomenclature, version 3a. Disponível em: http://www.ohio.edu/phylocode/ (Acessado em 27/06/2017).

Christoffersen M.L. (1995) Cladistic Taxonomy, Phylogenetic Systematics, and Evolutionary Ranking. Systematic Biology, 44(3): 440-454.

Coffey P. (1938) Ontology or the Theory of Being: An Introduction to General Metaphysics. Nova Iorque: Peter Smith. 439 p.

Darlington P.J. (1970) A practical criticismo of Hennig-Brundin "Phylogenetic Systematics" and Antarctic biogeography. Systematic Zoology, 19(1): 1-18.

Darwin C. (1859) On the Origin of Species by Means of Natural Selection, or the Preservation of Favoured Races in the Struggle for Life. Londres: John Murray. $502 \mathrm{p}$.

Dayrat B. (2003) The Roots of Phylogeny: How Did Haeckel Build His Trees? Systematic Biology, 52(4): 515-527.

De Pinna M.G.G. (1991) Concepts and tests of homology in the cladistics paradigm. Cladistics, 7: 367-394.

de Queiroz K. \& Gauthier J. (1990) Phylogeny as a central principle in taxonomy: Phylogenetic definitions of taxon names. Systematic Zoology, 39:307-322.

de Queiroz K. \& Gauthier J. (1992) Phylogenetic taxonomy. Annual Review of Ecology and Systematics, 23: 449-480.

de Queiroz K. \& Gauthier J. (1994) Toward a phylogenetic system of biological nomenclature. Trends in Ecology and Evolution, 9: 27-31.

de Queiroz K. \& Poe S. (2001) Philosophy and phylogenetic inference: A comparison of likelihood and parsimony methods in the context of Karl Popper's writings on corroboration. Systematic Biology, 50: 305-321.

Donoghue M.J. \& Kadereit J.W. (1992) Waler Zimmermann and the Growth of Phylogenetic Theory. Systematic Biology, 41(1): 74-85.

Farris J.A. (1983) The logical basis of phylogenetic analysis (p. 7-36). In: Platnick N.I. \& Funk V.A. (Eds). Advances in Cladistics. Volume 2. Nova Iorque: Columbia University Press. $250 \mathrm{p}$.

Farris J.S. (2008) Parsimony and explanatory power. Cladistics, 24: 825-847.

Farris J.S. (2013) Pattern taxonomy. Cladistics, 29: 228-229.

Farris J.S., Kluge A.G. \& Carpenter J.M. (2001) Popper and likelihood versus "Popper.*”. Systematic Biology, 50: 438-444.

Felsenstein J. (1981) Evolutionary trees from DNA sequences: a maximum likelihood approach. Journal of Molecular Evolution, 17: 368-376.

Felsenstein J. (2004) In ferring phylogenies. Sunderland: Sinauer Associates, Inc. 664 p.

Ferigolo J. (2015) A epistemologia de Aristóteles. Rio Grande do Sul: Editora Unisinos. 232 p.

Ferigolo J. (2016) Filosofia da Biologia de Aristóteles. Curitiba: Editora Prismas. 474 p.

Fitzhugh K. (2005a) Les bases philosophiques de l'inférence phylogénétique: une vue d'ensemble. In: Deleporte P. \& Lecointre G. (Eds). Philosophie de la systématique, Biosystema, 24: 83-105.

Fitzhugh K. (2005b) The inferential basis of species hypotheses: the solution to defining the term "species." Marine Ecology, 26: 155-165.

Fitzhugh K. (2006a) The abduction of phylogenetic hypotheses. Zootaxa, 1145: 1-110.

Fitzhugh K. (2006b) The philosophical basis of character coding for the inference of phylogenetic hypotheses. Zoologica Scripta, 35: 261-286. 
Fitzhugh K. (2006c) The 'requirement of total evidence' and its role in phylogenetic systematics. Biology and Philosophy, 21: 309-351.

Fitzhugh K. (2008a) Abductive inference: implications for 'Linnean' and 'phylogenetic' approaches for representing biological systematization. Evolutionary Biology, 35: 52-82.

Fitzhugh K. (2008b) Clarifying the role of character loss in phylogenetic inference. Zoologica Scripta, 37: 561-569.

Fitzhugh K. (2009) Species as explanatory hypotheses: refinements and implications. Acta Biotheoretica, 57: 201-248.

Fitzhugh K. (2010) Evidence for evolution versus evidence for intelligent design: parallel confusions. Evolutionary Biology, 37: 68-92.

Fitzhugh K. (2012) The limits of understanding in biological systematics. Zootaxa, 3435: 40-67.

Fitzhugh K (2014) Character mapping and cladogram comparison versus the requirement of total evidence: Does it matter for polychaete systematics? Memoires of Museum Victoria, 71: $67-78$.

Fitzhugh K. (2016a) Phylogenetic hypotheses: neither testable nor falsifiable. Evolutionary Biology, 43: 257-266.

Fitzhugh K. (2016b) Ernst Mayr, causal understanding, and systematics: an example using sabelliform polychaetes. Invertebrate Biology, 135: 302-313.

Fitzhugh K. (2016c) Sequence Data, Phylogenetic Inference, and Implications of Downward Causation. Acta Biotheoretica, 64(2): 133-60.

Fitzhugh K. (2016d) Dispelling five myths about hypothesis testing in biological systematics. Organisms Diversity \& Evolution, 16(3): 443-465.

Giribet G. (2015) Morphology should not be forgotten in the era of genomics-a phylogenetic perspective. Zoologischer Anzeiger, 256: 96-103.

Goloboff P.A. (2003) Parsimony, likelihood, and simplicity. Cladistics, 19: 91-103.

Griffiths G.C.D. (1974) On the foundations of biological systematics. Acta Biotheoretica, 23: 85131.

Haber M. (2011) Phylogenetic inference (p. 231-242). In: Tucker A. (Ed.). A Companion to the Philosophy of History and Historiography. Malden: Wiley-Blackwell. 576 p.

Haeckel E. (1866) Generelle Morphologie der Organismen. Berlim: Reimer. 574 p.

Hartmann M. (1933) Allgemeine Biologie. Eine Einführung in die Lehre vom Leben, zweite Auflage. Jena: Gustav Fischer. 238 p.

Hempel C.G. (1965) Aspects of scientific explanation and other essays in the philosophy of science. Nova Iorque: The Free Press. 504 p.

Hennig W. (1950) Grundzüge einer Teorie der phylogenetischen Systematik. Berlim: Deutscher Zentralverlag. $370 \mathrm{p}$.

Hennig W. (1965) Phylogenetic systematics. Annual Review of Entomology, 10: 97-116.

Hennig W. (1966) Phylogenetic systematics. Urbana: University of Illinois Press. 263 p.

Hennig W. (1968) Elementos de una sistemática filogenética. Buenos Aires: EUDEBA. 353 p.

Huelsenbeck J.P. \& Ronquist F. (2001) MrBayes: bayesian inference of phylogeny. Bioinformatics, 17: 754-755.

Huelsenbeck J.P., Ronquist F., Nielsen R. \& Bollback J.P. (2001) Bayesian inference of phylogeny and its impact on evolutionary biology. Science, 294: 2310-2314.

Joshi J. \& Edgecombe G.D. (2017) Tracking the variability of phenotypic traits on a molecular phylogeny: an example from scolopendrid centipedes in peninsular India. Organisms Diversity \& Evolution, 17(2): 393-408.

Kitching I.J., Forey P.L., Humphries C.J. \& Williams D.M. (1998) Cladistics: The Theory and Practice of Parsimony Analysis. The Systematics Association Publication No. 11. New York: Oxford University Press. 228 p.

Laland K.N., Sterelny K., Odling-Smee J., Hoppitt W. \& Uller T. (2011) Cause and Effect in Biology Revisited: Is Mayr's Proximate-Ultimate Dichotomy Still Useful? Science, 334(6062): $1512-1516$. 
Lankester E.R. (1870) On the use of the term homology in modern zoology, and the distinction between homogenetic and homoplastic agreements. Annals and Magazine of Natural History, 6: 35-43.

Lee M.S.Y. \& Palci A. (2015) Morphological Phylogenetics in the Genomic Age. Current Biology, 25: R922-R929.

Mahner M. \& Bunge M. (1997) Foundations of Biophilosophy. Nova Iorque: Springer-Verlag. 423 p.

Mayr E. (1961) Cause and effect in biology. Science, 131: 1501-1506.

Mayr E. (1969) Principles of systematic zoology. Nova Iorque: McGraw-Hill. 428 p.

Mayr E. (1974) Cladistic analysis and cladistics classification. Journal of Zoological Systematics and Evolutionary Research, 12(1): 94-128.

Michener C.D. \& Sokal R. (1957) A quantitative approach to a problem in classification. Evolution, 11(2): 130-162.

Nixon K.C. \& Carpenter J.M. (1993) On Outgroups. Cladistics, 9: 413-426.

Nixon K.C. \& Carpenter J.M. (2012) On homology. Cladistics, 28: 160-169.

O'Hara R.J. (1993) Systematic generalization, historical fate, and the species problem. Systematic Biology, 42: 231-246.

O'Hara R.J. (1997) Population thinking and tree thinking in systematics. Zoologica Scripta, 26: 323-329.

Omland K.E., Cook L.G. \& Crisp M.D. (2008) Tree thinking for all biology: the problem with reading phylogenies as ladders of progress. BioEssays, 30: 854-867.

O’Reilly J.E., Puttick M.N., Parry L., Tanner A.R., Tarver J.E., Fleming J., Pisani D. \& Donoghue P.C.J. (2016) Bayesian methods outperform parsimony but at the expense of precision in the estimation of phylogeny from discrete morphological data. Biology Letters, 12: 20160081.

Owen R. (1847) Report on the archetype and homologies of the vertebrate skeleton. Report on the Meeting of the British Association for the Advancement of Science, 16: 169-340.

Page R.D.M. \& Charleston M.A. (1998) Trees within trees: phylogeny and historical associations. Tree, 13(9): 356-359.

Page R.D.M. \& Holmes E.C. (1998) Molecular Evolution: A Phylogenetic Approach. Oxford: Blackwell Science. 346 p.

Patterson C. (1994) Null or minimal models (p. 173-192). In: Scotland R.W. \& Siebert D.J. (Eds). Models In Phylogeny Reconstruction. Oxford: Clarendon Press. 376 p.

Peirce C.S. (1878) Illustrations of the logic of science. Sixth paper. - Deduction, induction, and hypothesis. Popular Science Monthly, 13: 470-482.

Platnick N.I. (1979) Philosophy and the transformation of cladistics. Systematic Zoology, 28: 537-546.

Popper K.R. (1968) Conjectures and Refutations: The Growth of Scientific Knowledge. Nova Iorque: Harper \& Row. 417 p.

Popper K.R. (1992) Realism and the aim of science. Nova Iorque: Routledge. 464 p.

Rieppel O. (2003) Semaphoronts, cladograms and the roots of total evidence. Biological Journal of the Linnean Society, 80: 167-186.

Rieppel O. (2016) Willi Hennig as philosopher The Future of Phylogenetic Systematics: The Legacy of Willi Hennig (p. 356-376). In: Williams D., Schmitt M. \& Wheeler Q. (Eds). The Future of Phylogenetic Systematics: The Legacy of Willi Hennig. Londres: Cambridge University Press. 508 p.

Sereno P.C. (1999) Definitions in phylogenetic taxonomy: Critique and rationale. Systematic Biology, 48: 329-351.

Sereno P.C. (2005) The logical basis of phylogenetic taxonomy. Systematic Biology, 54: 595-619.

Sereno P.C. (2007) Logical basis for morphological characters in phylogenetics. Cladistics, 23: $565-587$.

Simakov O., Kawashima T., Marlétaz F., Jenkins J., Koyanagi R., Mitros T. Hisata K., Bredeson J., Shoguchi E., Gyoja F., Yue J., Chen Y., Freeman R.M., Sasaki A., Hikosaka-Katayama T., Sato A., Fujie M., Baughman K.W., Levine J., Gonzalez P., Cameron C., Fritzenwanker J.H., Pani 
A.M., Goto H., Kanda M., Arakaki N., Yamasaki S., Qu J., Cree A., Ding Y., Dinh H.H., Dugan S., Holder M., Jhangiani S.N., Kovar C.L., Lee S.L., Lewis L.R., Morton D., Nazareth L.V., Okwuonu G., Santibanez J., Chen R., Richards S., Muzny D.M., Gillis A., Peshkin L., Wu M., Humphreys T., Su Y., Putnam N.H., Schmutz J., Fujiyama A., Yu J., Tagawa K., Worley K.C., Gibbs R.A., Kirschner M.W., Lowe C.J., Satoh N., Rokhsar D.S. \& Gerhart J. (2015) Hemichordate genomes and deuterostome origins. Nature, 527: 459-465.

Sober E. (1988) Reconstructing the Past: Parsimony, Evolution, and Inference. Massachusetts: MIT Press, Cam- Zootaxa bridge. 265 p.

Sokal R.R. \& Sneath P.H.A. (1963) Principles of Numerical Taxonomy. San Francisco: W.H. Freeman and Company. 359 p.

Stevens P.F. (1994) The development of Biological Systematics. Nova Iorque: Columbia University Press. $617 \mathrm{p}$.

Swofford D.L., Olsen G.J., Waddell P.J. \& Hillis D.M. (1996) Phylogenetic inference (p. 407-514). In: Hillis D.M., Moritz C. \& Mable B.K. (Eds). Molecular Systematics. Massachusetts: Sinauer Associates. $655 \mathrm{p}$.

Wiens J.J. (2008) Systematics and Herpetology in the Age of Genomics. BioScience, 58(4): 297307.

Wiens J.J. (2009) Paleontology, Genomics, and Combined-Data Phylogenetics: Can Molecular Data Improve Phylogeny Estimation for Fossil Taxa? Systematic Biology, 58(1): 87-99.

Williams D.M. \& Ebach M.C. (2008) Foundations of Systematic and Biogeography. Berlim: Springer. $309 \mathrm{p}$.

Williams D.M. \& Ebach M.C. (2012) Confusing homologs as homologies: a reply to on homology. Cladistics, 28: 223-224.

Xu X. \& Pol D. (2003) Archaeopteryx, paravian phylogenetic analyses, and the use of probabilitybased methods for palaeontological datasets. Journal of Systematic Palaeontology, 12(3): 323-334.

Zachos F.E. (2016) Tree thinking and species delimitation: Guidelines for taxonomy and phylogenetic terminology. Mammalian Biology, 81: 185-188.

Ziehen T. (1934) Erkenntnistheorie. Zweite Auflage. Erster Teil. Allgemeine Grundlegung der Erkenntnistheorie. Spezielle Erkenntnistheorie der Empfndungstatsachen einschliesslich Raumtheorie. Jena: Gustav Fischer. 958 p.

Zimmerman W. (1931) Arbeitsweise der botanischen phylogenetic und anderer Gruppoerungwissenschaften (p. 941-1053). In: Abderhalden E. (Ed.). Handbuch der biologischen Arbeitsmethoden. Berlim: Urban \& Scwarzenber. 1053 p.

Zimmerman W. (1943) Die Methoden der Phylogenetik (p. 20-56). In: Heberer G. (Ed.). Die Evolution der Organismen. Stuttgart: 1. Aufl. G. Fischer, Jena, Germany. 661 p.

Zimmerman W. (1953) Evolution. Geschichte ihrer probleme und erkenntnisse. Berlim: Freiburg und Müchen. $623 \mathrm{p}$. 